\title{
Desunificação Nominal via Ponto Fixo
}

\author{
Leonardo Batista $^{1}$, Daniele Nantes-Sobrinho ${ }^{1}$ \\ ${ }^{1}$ Departamento de Matemática - Universidade de Brasília (UnB) \\ Brasília- Brasil \\ L.M.Batista@mat.unb.br, dnantes@unb.br
}

\begin{abstract}
This is a work-in-progress about solving equations and disequations between nominal terms, called the nominal disunification problem. Instead of using the standard nominal techniques that use freshness constraints ( i.e., expressions of the form a\#t that means "atom a is fresh for the nominal term $t$ ") to implement $\alpha$-equivalence, we follow an alternative approach that involves fixed-point equations, that is, equations of the form $\pi \cdot t \approx_{\alpha}^{?} t$, where $t$ is a nominal term and $\pi$ is a permutation. This approach will be convenient for future extensions of the problem involving equational theories.
\end{abstract}

Resumo. Este é um trabalho em andamento sobre a resolução de equações $e$ diferenças entre termos nominais, chamado de problema de desunificação nominal. Ao invés de usarmos as técnicas nominais padrões que envolvem restrições de freshness (i.e., expressões da forma a\#t que significa "o átomo a não ocorre livre em t”) para implementar $\alpha$-equivalência, seguiremos uma abordagem alternativa que envolve equações de ponto fixo, i.e., equações da forma $\pi \cdot t \approx_{\alpha}^{?} t$, onde té um termo nominal e $\pi$ uma permutação. Essa abordagem será conveniente a futuras extensões do problema envolvendo teorias equacionais.

\section{Introdução}

Este trabalho trata do problema de resolver equações $\left(s \approx_{\alpha}^{?} t\right)$ e diferenças/disequações $\left(s \approx_{\alpha} t\right)$ entre termos nominais, conhecido como o problema da desunificação nominal [Ayala-Rincón et al. 2020b], que tem a forma $\left\langle s \approx_{\alpha}^{?} t, u \not_{\alpha}^{?} v\right\rangle$, e consiste em decidir se existe uma substituição $\sigma$ tal que $s \sigma \approx_{\alpha} t \sigma$ mas $u \sigma \not_{\alpha} v \sigma$. Por exemplo, a substituição $\sigma=\{Z \mapsto \mathrm{f}(W)\}$ é uma solução para o problema de desunificação nominal $\left\langle\forall x P(x, Z) \approx_{\alpha}^{?} \forall y P(y, f(W)), \exists x \mathrm{f}(x) \not_{\alpha}^{?} \mathrm{f}(Z)\right\rangle$, onde $\mathrm{f}$ é um símbolo de função unário, $P$ é um predicado binário, $x, y$ são variáveis ligadas e $Z, W$ são variáveis livres. Basta observar que trocando $Z$ por $\mathrm{f}(W)$ faz os dois lados da equação iguais (módulo renomeamento das variáveis ligadas), e $\exists x \mathrm{f}(x) \not \varpi_{\alpha} \mathrm{f}(\mathrm{f}(W))$.

Técnicas nominais são úteis para o tratamento de linguagens que envolvem ligadores [Gabbay and Pitts 2002]. Nessa abordagem, ligações são implementadas através da abstração de átomos, e o renomeamento deles, através de permutações. Para isto, são considerados os predicados de freshness (que tem a forma $a \# t$ ) e $\alpha$-equivalência (que tem a forma $s \approx_{\alpha} t$ ). Intuitivamente, $a \# t$ significa que o átomo $a$ não pode ocorrer livre no termo $t$. Este conceito foi formalizado em [Pitts 2013] utilizando o quantificador new (И) que, na lógica nominal quantifica sobre nomes novos. Tal formalização é expressa pela seguinte sentença: $a \# x \Leftrightarrow\left(И a^{\prime}\right)\left(a a^{\prime}\right) \cdot x=x$, isto é, $a$ ocorre ligado em $x$ se, e somente se, para qualquer átomo novo $a^{\prime}$, a permutação $\left(a a^{\prime}\right)$ fixa $x$. Por exemplo, considere a 
fórmula $\phi=\forall a P(a)$. Nesse caso, $a$ é uma variável que ocorre ligada pelo quantificador $\forall$, portanto $a \# \phi$, o que equivale a dizer que o renomeamento de $a$ por uma variável nova ainda preserva $\phi$, ou seja, $\left(И a^{\prime}\right)\left(a a^{\prime}\right) \cdot \phi(a) \approx_{\alpha} \phi(a)$.

Em [Ayala-Rincón et al. 2020a] foi proposta uma nova axiomatização para a relação de $\alpha$-equivalência em termos nominais utilizando pontos fixos, isto é, expressões da forma $\pi \cdot t \stackrel{\hat{\approx}}{\alpha} t$ (lê-se: "a permutação $\pi$ fixa o termo $t$ ”). Ou seja, ao invés de utilizarmos os predicado de freshness que absorve o ponto fixo, exploramos esta última propriedade, e tratamos de uma linguagem puramente equacional. Em unificação nominal o uso da relação de freshness e de ponto fixo coincidem, mas é na presença de teorias equacionais que essas abordagens se diferem, e o método via ponto fixo se destaca.

Observamos o caso $\approx_{\alpha, \mathrm{C}}(\alpha$-equivalência módulo comutatividade): por exemplo, uma equação do tipo $(a b) \cdot X \approx_{\alpha, \mathrm{C}}^{\text {? }} X$, usando a abordagem usual de freshness, teria uma solução $\langle a, b \# X, \sigma\rangle$ (qualquer substituição $\sigma$ desde que $a, b$ não ocorram livres em $X \sigma)$ [Urban et al. 2004]. Observe que as substituições $\{X \mapsto f(e)\},\{X \mapsto \forall a(P(a) \vee$ $Q(a))\}, \ldots$ são soluções, no entanto, infinitas soluções $(\{X \mapsto a+b\},\{X \mapsto(a+b)+(a+$ $b)\},\{X \mapsto \mathrm{f}(a+b)\}, \ldots)$ são perdidas. De fato, note que $(a b) \cdot(a+b)=b+a \approx_{\alpha, \mathrm{c}} a+b$, logo a permutação $(a b)$ fixa o termo $a+b$, apesar dos átomos $a$ e $b$ ocorrerem livres em $a+b$. Com a abordagem de ponto fixo, a completude do algoritmo de unificação nominal (módulo comutatividade) é recuperada [Ayala-Rincón et al. 2020b].

Recentemente, em [Ayala-Rincón et al. 2020b], foi proposto um método para decidir o problema da desunificação nominal, que trata-se de uma extensão do problema de desunificação de primeira-ordem definido em [Buntine and Bürckert 1994, Comon and Lescanne 1989], onde a relação de $\alpha$-equivalência é construída usando permutações e freshness. No entanto, o algoritmo de decisão para desunificação nominal proposto depende de que o problema de unificação nominal correspondente seja finitário. Enquanto que o problema de unificação nominal (puro) é finitário [Urban et al. 2004], quando teorias equacionais são envolvidas, esta propriedade é perdida, como foi provado em [Ayala-Rincón et al. 2017].

Contribuições. Neste trabalho definimos o problema de desunificação nominal via ponto fixo (Definição 4.1) e estendemos para esta abordagem vários conceitos necessários para o estudo da sua decidibilidade: uma nova noção de solução para esse problema (Definição 4.4) que depende do conceito de par com exceções (Definição 4.2) bem como sua consistência (Definição 4.3). Provamos resultados de consistência (Corolário 4.1), apresentamos o algoritmo (Algoritmo 2) para obter um conjunto completo de soluções (Teorema 4.1). Este é um primeiro passo para o desenvolvimento de extensões do problema de desunificação nominal que envolvam teorias equacionais.

Organização. A Seção 2 contém noções básicas de sintaxe nominal bem como as construções via ponto fixo. A Seção 3 introduz o problema da unificação nominal via ponto fixo bem como o algoritmo de decisão. Na Seção 4 introduzimos o conceito de desunificação nominal via ponto fixo, bem como os resultados obtidos com esta técnica. Finalmente, na Seção 5 apresentamos a conclusão e discutimos o trabalho futuro.

\section{Preliminares}

Seja $\mathbb{A}$ um conjunto infinito e enumerável de elementos $a, b, c, \ldots$, que serão chamados átomos (ou nomes atômicos) e $\mathcal{X}=\{X, Y, Z, \ldots\}$ um conjunto infinito enumerável de variáveis. Fixamos um conjunto finito de símbolos de função $\mathcal{F}=\{\mathrm{f}, \mathrm{g}, \ldots\}$ - disjunto 
de $\mathbb{A}$ e $\mathcal{X}$ - tal que cada $\mathrm{f} \in \mathcal{F}$ é associado a um único inteiro não-negativo $n$ tal que $\mathrm{f}: n$, a sua aridade. Uma permutação $\pi$ em $\mathbb{A}$ é uma bijeção em $\mathbb{A}$ com domínio finito, isto é, $\operatorname{dom}(\pi)=\{a \in \mathbb{A} \mid \pi(a) \neq a\}$ é finito. Escrevemos $I d$ para permutação identidade, denotamos por $\pi^{-1}$ a permutação inversa de $\pi$ e $\pi \circ \pi^{\prime}$ denota a composição de $\pi$ e $\pi^{\prime}$.

Termos nominais são gerados pela gramática: $t:=a|[a] t| \mathrm{f}\left(t_{1}, \ldots, t_{n}\right) \mid \pi \cdot X$, onde $a$ é um átomo, $[a] t$ denota a abstração do átomo $a$ no termo $t, f\left(t_{1}, \cdots, t_{n}\right)$ denota a aplicação de $\mathrm{f}$ em termos $t_{1}, \ldots, t_{n}, \operatorname{com} \mathrm{f} \in \mathcal{F}$ e $\mathrm{f}: n$, e $\pi \cdot X$ é uma variável moderada ou uma suspensão, onde $\pi$ é uma permutação de átomos. Abreviaremos a sequência ordenada $t_{1}, \ldots, t_{n}$ por $(\widetilde{t})_{n}$. Denotamos por $\operatorname{Var}(t)$ o conjunto das variáveis que ocorrem em $t$. Termos básicos são aqueles sem ocorrência de variáveis, ou seja, $\operatorname{Var}(t)=\emptyset$.

As permutações de átomos serão representadas por listas finitas de transposições, cuja notação $(a b)$ indica a troca de $a$ por $b$ e vice-versa. Assim, uma permutação $\pi$ é gerada pela gramática: $\pi:=I d \mid(a b) \circ \pi$, onde $I d$ é geralmente omitida na representação de uma permutação $\pi$. A ação de uma permutação $\pi \mathrm{em}$ um termo $t$ é definida indutivamente por: $\pi \cdot a=\pi(a), \pi \cdot\left(\pi^{\prime} \cdot X\right)=\left(\pi \circ \pi^{\prime}\right) \cdot X, \pi \cdot([a] t)=[\pi(a)](\pi \cdot t) \mathrm{e}$ $\pi \cdot \mathrm{f}\left(t_{1}, \ldots, t_{n}\right)=\mathrm{f}\left(\left(\pi \cdot t_{1}, \ldots, \pi \cdot t_{n}\right)\right.$. Uma variável moderada da forma $I d \cdot X$ será escrita apenas como $X$. Substituições são geradas pela gramática: $\sigma:=i d \mid[X \mapsto s] \sigma$. A ação de uma substituição em um termo $t$, denotada por $t \sigma$, é definida indutivamente por: $a \sigma=a,(\pi \cdot X) \sigma=\pi \cdot(X \sigma),([a] t) \sigma=[a](t \sigma)$ e $\left(\mathrm{f}\left(t_{1}, \ldots, t_{n}\right)\right) \sigma=\mathrm{f}\left(t_{1} \sigma, \ldots, t_{n} \sigma\right) . \mathrm{O}$ símbolo ○ também será utilizado para composição de substituições: $t\left(\sigma \circ \sigma^{\prime}\right)=(t \sigma) \sigma^{\prime}$. Substituições e permutações comutam [Fernández and Gabbay 2007]: $\pi \cdot(s \sigma)=(\pi \cdot s) \sigma$.

\subsection{Suporte}

Seja $S$ um conjunto equipado com uma ação do grupo $\operatorname{Perm}(\mathbb{A})$, o grupo das permutações finitas de $\mathbb{A}$. Um conjunto $A \subset \mathbb{A}$ é um suporte para um elemento $x \in S$ se para todo $\pi \in \operatorname{Perm}(\mathbb{A})$, vale $((\forall a \in A) \pi(a)=a) \Rightarrow \pi \cdot x=x$. Um conjunto nominal $S$ é um conjunto equipado com uma ação do grupo $\operatorname{Perm}(\mathbb{A})$ e que todos os seus elementos possuem suporte finito. Denotamos por $\operatorname{supp}_{S}(x)$ o menor suporte finito de $x \in S$, que coincide com $\operatorname{supp}_{S}(x):=\bigcap\{A \in \mathcal{P}(\mathbb{A}) \mid A$ é um suporte finito para $x\}$, como mostrado em [Pitts 2013]. Escrevemos $\operatorname{supp}(x)$ quando $S$ está claro pelo contexto.

Note que $\mathbb{A}$ é um conjunto nominal: sob a ação natural de $\operatorname{Perm}(\mathbb{A})$, cada $a \in \mathbb{A}$ tem suporte finito dado pelo conjunto unitário que o contém $\{a\}$, portanto $\operatorname{supp}(a)=\{a\}$. Exemplo 2.1. Para determinar o suporte de uma permutação $\pi$, relembramos que $\operatorname{Perm}(\mathbb{A})$ age sobre si mesmo por conjugação, onde a noção de conjugação é a seguinte: dados $a$ e $b$ elementos de um grupo $G$, dizemos que $b$ é o conjugado de a se existe $g \in G$ tal que $g a g^{-1}=b$. No caso de $\operatorname{Perm}(\mathbb{A})$, o conjugado de $\pi$ com respeito a $\rho$ é a composição $\rho \circ \pi \circ \rho^{-1}$ denotada por $\pi^{\rho}$, e será o resultado da ação $\rho \cdot \pi$. Segue da definição suporte que $\operatorname{supp}(\pi)=\operatorname{dom}(\pi)$. $\operatorname{Logo} \operatorname{Perm}(\mathbb{A})$ é um conjunto nominal.

\subsection{Ponto Fixo para $\alpha$-equivalência}

Pontos fixos de uma permutação terão um papel importante na definição de $\alpha$ equivalência entre termos nominais. Definimos, então, a relação de ponto fixo.

Definição 2.1 (Ponto Fixo). Seja $S$ um conjunto nominal. A relação de ponto fixo $\curlywedge \subseteq$ $\operatorname{Perm}(\mathbb{A}) \times S$ é definida como: $\pi \curlywedge x \Leftrightarrow \pi \cdot x=x$. Lê-se " $\pi$ fixa $x$ ".

Observamos que se $\operatorname{dom}(\pi) \cap \operatorname{supp}(x)=\emptyset$, então $\pi \curlywedge x$ vale, o que segue diretamente da definição de suporte. Entretanto, a contrapartida dessa implicação não é válida 
em geral. Por exemplo, considere expressões construídas usando átomos e um operador binário comutativo +; a permutação $\pi=(a b)$ fixa a classe de equivalência módulo comutatividade do termo nominal $a+b$, isto é, fixa os termos em $\{a+b, b+a\}$, apesar do fato de seu suporte coincidir com $\operatorname{dom}(\pi)$.

Definição 2.2. Uma restrição de ponto fixo é um par $\pi \curlywedge t$ de uma permutação $\pi$ e um termo $t$. Uma restrição de $\alpha$-equivalência é um par de termos da forma $s \hat{\approx}_{\alpha} t$.

Considerando $s$ e $t$ termos básicos, a restrição $s \stackrel{\curlywedge}{\alpha}_{\alpha} t$ significa que $s$ e $t$ são $\alpha$ equivalentes, ou seja, equivalentes módulo renomeamento de átomos abstraídos/ligados. Já a restrição $\pi \curlywedge t$ significa que a permutação $\pi$ fixa o termo $t$, isto é, $\pi \cdot t \hat{\approx}_{\alpha} t$. Em outras palavras, $\pi$ não tem efeito sobre $t$ exceto pelo renomeamento de átomos ligados, por exemplo, $(a b) \curlywedge[a] \mathrm{g}(a, \mathrm{~g}(c, d))$ mas não $(a b) \curlywedge \mathrm{g}(a, \mathrm{~g}(c, d))$. No caso de termos não-básicos, ambas as restrições precisam ser avaliadas sob um contexto, que fornecerá informações sobre permutações que fixam variáveis.

Um contexto (de ponto fixo) é um conjunto finito de restrições primitivas de ponto fixo, isto é, restrições da forma $\pi \curlywedge X$ ou $\pi \curlywedge a$. Usaremos as letras maiúsculas $\Upsilon, \Psi, \Phi, \Delta, \ldots$ para representar contextos. Um contexto é consistente quando não possui restrições do tipo $\pi \curlywedge a$, onde $a \in \operatorname{dom}(\pi)$. Um sequente de ponto fixo (resp. de $\alpha$-equivalência) é um par $\Upsilon \vdash \pi \curlywedge t$ composto por um contexto consistente $\Upsilon$ e uma restrições de ponto fixo (resp. de $\alpha$-equivalência). Dizemos que $\Upsilon$ ou $\Psi$ implicam essas restrições, ou que as restrições são deriváveis dos respectivos contextos. Se uma restrições $C$ não pode ser derivada do contexto $\Delta$, escrevemos $\Delta \forall C$. Essa noção de implicação é naturalmente extendida para conjuntos de restrições. Os sequentes de ponto fixo e $\alpha$-equivalência válidos são definidos pelas regras de dedução presentes na Tabela 1. Notações nas regras $(\curlywedge \mathbf{v})$ e $\left(\hat{\approx}_{\alpha} \mathbf{v}\right): \operatorname{supp}\left(\operatorname{perm}\left(\left.\Upsilon\right|_{X}\right)\right):=\cup_{\pi \in \operatorname{perm}\left(\left.\Upsilon\right|_{X}\right)} \operatorname{supp}(\pi)$, onde $\operatorname{perm}\left(\left.\Upsilon\right|_{X}\right)=\{\pi \mid \pi \curlywedge X \in \Upsilon\} ;$ e $\pi \curlywedge \operatorname{Var}(t):=\{\pi \curlywedge X \mid X \in \operatorname{Var}(t)\}$.

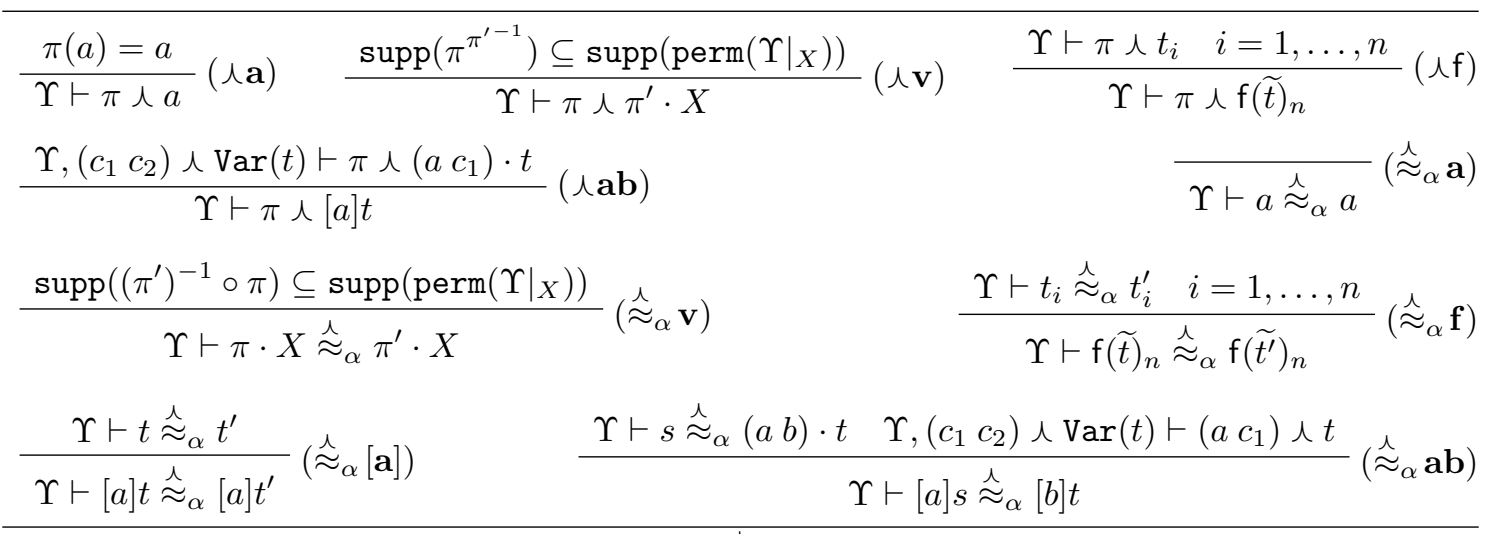

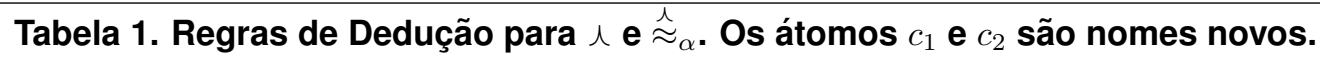

A seguir descrevemos as regras mais interessantes: na regra $(\curlywedge \mathbf{v})$ a condição $\operatorname{supp}\left(\pi^{\pi^{\prime-1}}\right) \subseteq \operatorname{supp}\left(\operatorname{perm}\left(\left.\Upsilon\right|_{X}\right)\right)$ impõe que a permutação $\pi^{\pi^{\prime-1}}$ tenha efeito apenas em átomos já afetados pelas permutações que fixam $X$. Com isso, a permutação em questão também fixará a variável. A regra $\left(\approx_{\alpha}\right.$ ab) estabelece que para duas abstrações $[a] t$ e $[b] s$ serem equivalentes, devemos obter duas condições: os termos $s$ e $t$ devem ser equivalentes se renomearmos em $t$ o átomo abstraído $b$ por $a$; e, além disso, a troca de $a$ por um átomo novo $c_{1}$ deve fixar $t$, garantindo assim a ocorrência ligada de $a$ também em $t$. Nas regras 


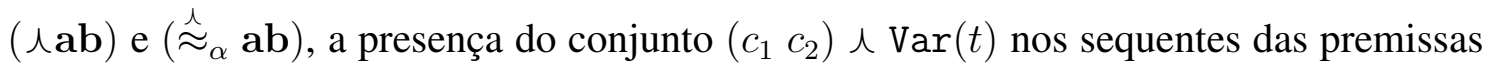
tem por objetivo certificar que $c_{1}$ seja realmente um nome atômico novo.

Exemplo 2.2. O termo $[a] \mathrm{f}(a)$ é fixado pela permutação $(a b)$, visto que $(a b) \cdot[a] \mathrm{f}(a)=$ $[b] \mathrm{f}(b)$, que é $\alpha$-equivalente a $[a] \mathrm{f}(a)$. Podemos derivar o sequente $\vdash(a b) \curlywedge[a] \mathrm{f}(a)$. Entretanto, não podemos deduzir $\vdash(a b) \curlywedge \mathrm{f}(a)$, e $\mathrm{f}(a)$ não é ponto fixo de $(a b)$, como esperado.

\section{Unificação Nominal via Ponto Fixo}

Esta seção trata de unificação nominal via ponto fixo ([Ayala-Rincón et al. 2020a]), que consiste em verificar se, dados dois termos nominais $s$ e $t$, existe uma substituição $\sigma$ tal que $s \sigma \stackrel{\curlywedge}{\approx}_{\alpha} t \sigma$, que difere da noção usual de unificação nominal [Urban et al. 2004].

Definição 3.1. Um problema de unificação nominal $\operatorname{Pr}$ é um $\operatorname{par} \operatorname{Pr}=(\Upsilon, P)$ onde $\Upsilon$ é um contexto consistente e $P$ consiste em um conjunto finito de restrições de igualdade e de ponto fixo da forma $s \stackrel{\imath}{?}_{\alpha}^{t} t$ e $\pi \iota^{?} t$, respectivamente.

Para contextos consistentes $\Upsilon$ e $\Psi$ e uma substituição $\sigma$, denotamos por $\Psi \sigma$ o conjunto $\{\pi \curlywedge X \sigma \mid \pi \curlywedge X \in \Psi\}$, e $\Upsilon \vdash \Psi \sigma$ denota $\Upsilon \vdash \pi \curlywedge X \sigma$, para todo $\pi \curlywedge X \in \Psi$. Definição 3.2. Uma solução para um problema $\operatorname{Pr}=(\Upsilon, P)$ é um $\operatorname{par}\langle\Phi, \sigma\rangle$ que satisfaz as seguintes condições: $(i) \Phi \vdash \Upsilon \sigma$; $(i i) \Phi \vdash \pi \curlywedge t \sigma$, se $\pi \curlywedge^{?} t \in P$; e $(i i i) \Phi \vdash s \sigma \stackrel{\varkappa}{\alpha}_{\alpha} t \sigma$, se $s \stackrel{\curlywedge}{*}_{\alpha}^{?} t \in P$.

$\mathcal{U}(\operatorname{Pr})$ denota o conjunto de soluções do problema $\operatorname{Pr}$. Soluções em $\mathcal{U}(\operatorname{Pr})$ podem ser comparadas usando a relação de ordem a seguir.

Definição 3.3. Sejam $\Phi_{1}, \Phi_{2}$ contextos consistentes de ponto fixo, e $\sigma_{1}, \sigma_{2}$ substituições. Dizemos que $\left\langle\Phi_{2}, \sigma_{2}\right\rangle$ é uma instância de $\left\langle\Phi_{1}, \sigma_{1}\right\rangle$, e denotamos $\left\langle\Phi_{1}, \sigma_{1}\right\rangle \leq\left\langle\Phi_{2}, \sigma_{2}\right\rangle$,

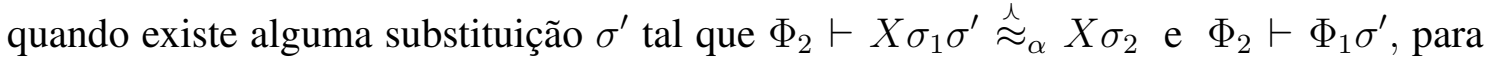
todo $X$. De modo mais específico, podemos escrever $\left\langle\Phi_{1}, \sigma_{1}\right\rangle \leq_{\sigma^{\prime}}\left\langle\Phi_{2}, \sigma_{2}\right\rangle$.

Definição 3.4. Uma solução principal (ou mais geral) para um problema Pr é o menor elemento de $\mathcal{U}(\operatorname{Pr})$ com relação $\mathrm{a} \leq$.

As regras de simplificação ${ }^{1}$ apresentadas na Tabela 2 implementam um algoritmo de unificação nominal (Unif): aplicamos as regras de simplificação em um problema Pr até que sua forma normal $\langle\mathrm{Pr}\rangle_{\mathrm{nf}}$ seja alcançada ${ }^{2}$. Escrevemos $\mathrm{Pr} \Longrightarrow \mathrm{Pr}^{\prime}$ quando $\mathrm{Pr}^{\prime}$ é obtido a partir de Pr aplicando uma regra de simplificação, e $\stackrel{*}{\Longrightarrow}$ denota o fecho reflexivotransitivo da relação $\Longrightarrow$. A terminação e confluência dessa relação são provadas em [Ayala-Rincón et al. 2020a]. Se $\operatorname{Pr} \stackrel{*}{\Longrightarrow} \operatorname{Pr}^{\prime}$ e $\operatorname{Pr}^{\prime}$ é irredutível (nenhuma regra pode ser aplicada), dizemos que $\operatorname{Pr}^{\prime}$ é uma forma normal de $\operatorname{Pr}$ e escrevemos $\operatorname{Pr}^{\prime}=\langle\operatorname{Pr}\rangle_{\mathrm{nf}}$.

$\mathrm{Se}\langle\operatorname{Pr}\rangle_{\mathrm{nf}}$ falha ou contém restrições equacionais reduzidas (que não podem ser simplificadas), dizemos que $\operatorname{Pr}$ é insolúvel; caso contrário, $\langle\operatorname{Pr}\rangle_{\mathrm{nf}}$ é solúvel e sua solução, denotada por $\langle\operatorname{Pr}\rangle_{\text {sol }}=\langle\Phi, \sigma\rangle$, é composta pela composição $\sigma$ de substituições aplicadas nos passos de simplificação e pelo contexto de ponto fixo $\Phi=\left\{\pi \curlywedge X \mid \pi \curlywedge^{?} X \in\langle\operatorname{Pr}\rangle_{\mathrm{nf}}\right\}$. Teorema 3.1 ([Ayala-Rincón et al. 2020a]). Seja Pr um problema de unificação, e suponha que $\langle\operatorname{Pr}\rangle_{\text {sol }}=\langle\Phi, \sigma\rangle$. Então: (1) $\langle\Phi, \sigma\rangle \in \mathcal{U}(\operatorname{Pr})$; e (2) $\langle\Phi, \sigma\rangle \leq\left\langle\Phi^{\prime}, \sigma^{\prime}\right\rangle$ para todo outro $\operatorname{par}\left\langle\Phi^{\prime}, \sigma^{\prime}\right\rangle \in \mathcal{U}(\operatorname{Pr})$. Isto é, $\langle\operatorname{Pr}\rangle_{\text {sol }}$ também é uma solução principal.

\footnotetext{
${ }^{1}$ Para um conjunto $S: \overline{\pi \curlywedge S}=\{\pi \curlywedge X \mid X \in S\}$.

${ }^{2}$ Em [Ayala-Rincón et al. 2020a], foi provado que este algoritmo retorna soluções idempotentes.
} 


$$
\begin{aligned}
& \text { (ノa) } \quad \operatorname{Pr} \uplus\left\{\pi \curlywedge^{?} a\right\} \quad \Longrightarrow \quad \operatorname{Pr} \text {, if } \pi(a)=a \\
& (\curlywedge f) \quad \operatorname{Pr} \uplus\left\{\pi \curlywedge ? \mathrm{f}(\widetilde{t})_{n}\right\} \quad \Longrightarrow \quad \operatorname{Pr} \cup\left\{\pi \lambda^{?} t_{1}, \ldots, \pi \curlywedge^{?} t_{n}\right\}
\end{aligned}
$$

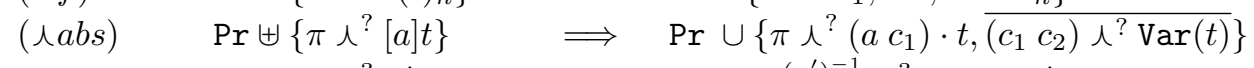

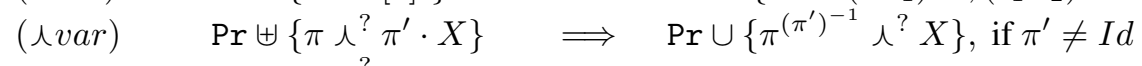

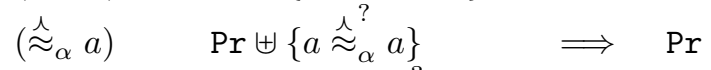

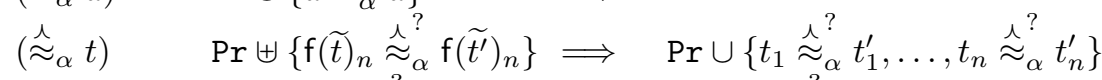

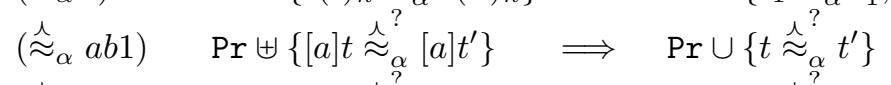

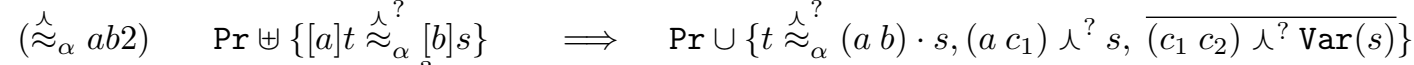

$$
\begin{aligned}
& \left(\stackrel{\jmath}{\approx}_{\alpha} \text { var }\right) \quad \operatorname{Pr} \uplus\left\{\pi \cdot X{\stackrel{\curlywedge}{\mathcal{\approx}_{\alpha}}}_{\alpha}^{?} \pi^{\prime} \cdot X\right\} \Longrightarrow \operatorname{Pr} \cup\left\{\left(\pi^{\prime}\right)^{-1} \circ \pi \curlywedge^{?} X\right\}
\end{aligned}
$$

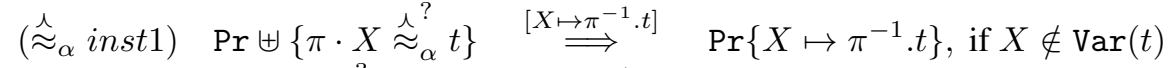

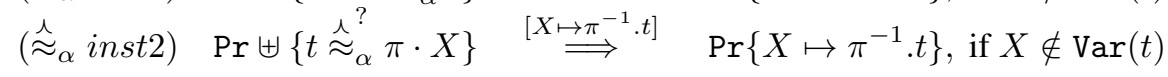

\section{Tabela 2. Regras de Simplificação. Os átomos $c_{1}$ e $c_{2}$ são nomes novos.}

\section{Desunificação Nominal via Ponto Fixo}

Esta seção contém as contribuições que estendem [Ayala-Rincón et al. 2020b]. A seguir, definimos o problema de desunificação nominal via ponto fixo.

Definição 4.1. Um problema de desunificação nominal $\mathcal{P}_{\curlywedge}$ é um par $\mathcal{P}_{\curlywedge}=\left\langle E_{\curlywedge} \| D_{\curlywedge}\right\rangle$,

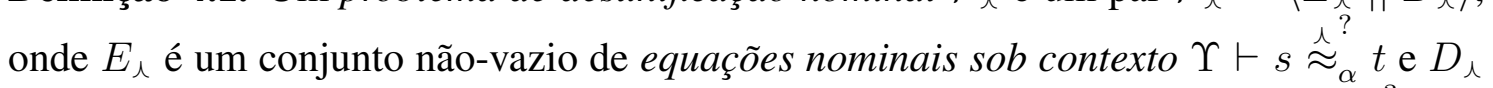
é um conjunto (possivelmente vazio) de diferenças nominais sob contexto $\Omega \vdash p \gtrless_{\alpha}^{\text {? }} q$ :

$$
E_{\curlywedge}=\left\{\Upsilon \vdash s_{1} \stackrel{\curlywedge}{*}_{\alpha}^{?} t_{1}, \ldots, s_{n} \stackrel{\curlywedge}{*}_{\alpha}^{?} t_{n},\right\}, D_{\curlywedge}=\left\{\Omega \vdash p_{1} \dot{z}_{\alpha}^{?} q_{1}, \ldots, p_{m} \ddot{z}_{\alpha}^{?} q_{m}\right\} .
$$

Os conjuntos $\Upsilon$ e $\Omega$ são contextos consistentes e serão chamados de condições iniciais de ponto fixo impostas ao problema $\mathcal{P}_{\curlywedge}$.

O próximo exemplo ilustra o que se espera de uma solução para um problema de desunificação nominal: um par $\langle\Phi, \sigma\rangle$ que será solução para a parte equacional do problema, porém, com a restrição de que ele não pode solucionar as equações associadas às diferenças presentes na outra parte.

Exemplo 4.1. Considere o problema $\mathcal{P}_{\curlywedge}=\left\langle\vdash X{\stackrel{\imath}{\approx_{\alpha}}}_{\alpha}(a b) \cdot Y \| \vdash[a] X{\underset{\neq}{\alpha}}_{\alpha}^{?}[b] Y\right\rangle . \quad \mathrm{A}$ equação associada a ele é $[a] X \stackrel{\imath}{\text { ? }}_{\alpha}[b] Y$. Usando a regra $\left(\stackrel{\curlywedge}{\approx}_{\alpha} a b 2\right)$ da Tabela 2 , temos: $\left\{[a] X \stackrel{\imath}{\approx}_{\alpha}^{p}[b] Y\right\} \Longrightarrow\left\{X \stackrel{\curlywedge}{\approx}_{\alpha}^{?}(a b) \cdot Y,\left(a c_{1}\right) \curlywedge^{?} Y,\left(c_{1} c_{2}\right) \curlywedge^{?} Y\right\}$. Se considerarmos um contexto sem adicionar as restrições $\left(a c_{1}\right) \curlywedge Y$ e $\left(\begin{array}{ll}c_{1} & \left.c_{2}\right)\end{array}\right) Y$, e escolhendo uma substituição de modo que a parte equacional seja satisfeita, a equação associada não será resolvida. Portanto, uma solução para $\mathcal{P}_{\curlywedge}$ será o $\operatorname{par}\langle\Phi, \sigma\rangle=\langle\emptyset,[X \mapsto(a b) \cdot Y]\rangle$.

Definição 4.2 (Par com exceções). Um par com exceções, denotado por $\langle\Phi, \sigma\rangle-\Theta$, consiste em um par $\langle\Phi, \sigma\rangle$ e uma família indexada de pares $\Theta=\left\{\left\langle\nabla_{l}, \theta_{l}\right\rangle \mid l \in I\right\}$.

A definição de par com exceções será importante para representar soluções de um problema de desunificação que tem restrições sobre como elas podem ser instanciadas. Por exemplo, se temos o problema $\left\langle\vdash X \stackrel{\imath}{\text { ₹ }}_{\alpha}^{\text {? }} Y \| \vdash X \mathcal{z}_{\alpha}^{?}[b] \mathrm{f}(b)\right\rangle$, as soluções para a equação $\vdash X \stackrel{\stackrel{*}{*}}{\text { ? }}_{\alpha} Y$ podem ser instanciadas livremente, com exceção das instâncias que levam $X$ para $[b] \mathrm{f}(b)$ ou termos $\alpha$-equivalentes. 
Definição 4.3. Dizemos que :

(i) um $\operatorname{par}\langle\Phi, \sigma\rangle$ é uma instância de uma família $\Theta=\left\{\left\langle\nabla_{l}, \theta_{l}\right\rangle \mid l \in I\right\}$ se, e somente se, toda instância de $\langle\Phi, \sigma\rangle$ é uma instância de algum $\left\langle\nabla_{l}, \theta_{l}\right\rangle \in \Theta$; e

(ii) um $\operatorname{par}\langle\Delta, \lambda\rangle$ é uma instância de um par com exceções $\langle\Phi, \sigma\rangle-\Theta$ se, e somente se, $\langle\Delta, \lambda\rangle$ é uma instância de $\langle\Phi, \sigma\rangle$ mas não de $\Theta$.

(iii) Um par com exceções $\langle\Phi, \sigma\rangle-\Theta$ é consistente se, e somente se, possui pelo menos uma instância.

Exemplo 4.2. Considere o problema $\mathcal{P}^{\prime}{ }_{\curlywedge}$, uma modificação do Exemplo 4.1: $\mathcal{P}_{\curlywedge}^{\prime}=\left\langle\left\{\left(a c_{1}\right) \curlywedge Y,\left(c_{1} c_{2}\right) \curlywedge Y\right\} \vdash X{\stackrel{\varkappa}{\approx_{\alpha}}}_{\alpha}^{?}(a b) \cdot Y \| \vdash[a] X \mathcal{z}_{\alpha}^{?}[b] Y\right\rangle$. Nesse caso não há solução, pois sempre que solucionamos a parte equacional, solucionamos também a equação associada à disequação. Obtemos, assim, par com exceções inconsistente: $\langle\Phi,[X \mapsto(a b) \cdot Y]\rangle-\{\langle\Phi,[X \mapsto(a b) \cdot Y]\rangle\}$, onde $\Phi=\left\{\left(a c_{1}\right) \curlywedge Y,\left(c_{1} c_{2}\right) \curlywedge Y\right\}$.

Lema 4.1 (Lema da Inconsistência). Um par com exceções $\langle\Phi, \sigma\rangle-\Theta$ é inconsistente se, e somente se, $\langle\Phi, \sigma\rangle$ é uma instância de $\Theta$.

O seguinte corolário nos permite construir um algoritmo para testar a consistência de pares com exceção (Algoritmo 1), uma vez que tenhamos resolvido, para todas as variáveis em $\mathcal{P}_{\curlywedge}$, o problema de matching-in-context $(\Phi \vdash X \sigma){\stackrel{\curlywedge}{{ }_{\alpha}}}_{\text {? }}(\Phi \vdash X \theta)$, onde $X \sigma$ é o lado que não pode ser instanciado (ver [Ayala-Rincón et al. 2020b]), cuja solução seria uma substituição $\delta$ tal que $\Phi \vdash X \sigma \stackrel{\curlywedge}{\approx}_{\alpha} X \theta \delta$.

Corolário 4.1. Seja $\langle\Phi, \sigma\rangle-\Theta$ um par com exceções. Se há um par $\left\langle\nabla_{l}, \theta_{l}\right\rangle \in \Theta$ tal

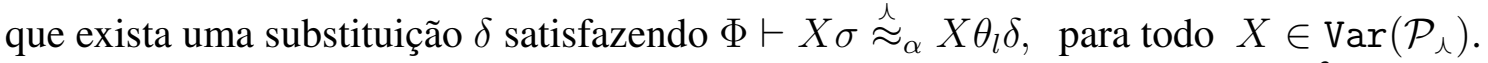
Então, $\langle\Phi, \sigma\rangle-\Theta$ é inconsistente sse $\operatorname{supp}\left(\operatorname{Perm}\left(\left\langle\nabla_{l} \delta\right\rangle_{\mathrm{nf}}\right)\right) \subseteq \operatorname{supp}(\operatorname{Perm}(\Phi))^{3}$.

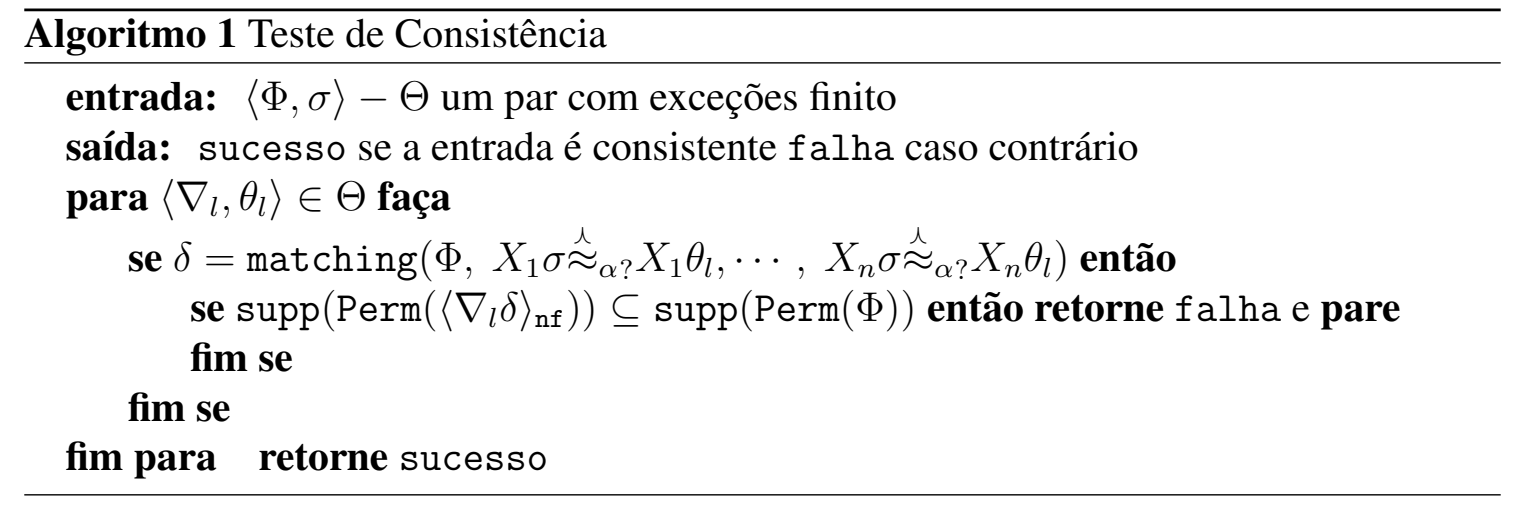

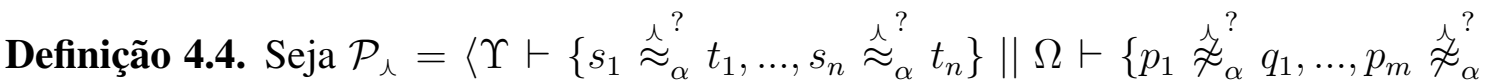
$\left.\left.q_{m}\right\}\right\rangle$ um problema de desunificação nominal. Uma solução para $\mathcal{P}_{\curlywedge}$ é um $\operatorname{par}\langle\Phi, \sigma\rangle$ onde $\Phi$ é um contexto consistente e $\sigma$ uma substituição satisfazendo as seguintes condições: (i) $\langle\Phi, \sigma\rangle$ é uma solução da parte equacional $E_{\curlywedge}$ de $\mathcal{P}_{\curlywedge}$; (ii) $\langle\Phi, \sigma\rangle$ satisfaz as diferenças em $D_{\curlywedge}$ de $\mathcal{P}_{\curlywedge}$, isto é: a) $\Phi \nvdash \Omega \sigma$, ou $\quad$ b) $\Phi \not p \sigma \approx_{\alpha} q \sigma$, para toda $p \not_{\alpha}^{\text {? }} q$ em $D_{\curlywedge}$.

Definição 4.5. Dizemos que um conjunto $S$ de pares com exceções é uma representação completa das soluções para o problema $\mathcal{P}_{\curlywedge}$ se, e somente se, $S$ satisfaz as seguintes condições: (i) se $\langle\Phi, \sigma\rangle-\Theta \leq\langle\Delta, \lambda\rangle$ para algum $\langle\Phi, \sigma\rangle-\Theta$ em $S$, então $\langle\Delta, \lambda\rangle$ é solução para $\mathcal{P}_{\curlywedge}$; (ii) se o par $\langle\Delta, \lambda\rangle$ é solução para $\mathcal{P}_{\curlywedge}$, então ele é uma instância de algum $\langle\Phi, \sigma\rangle-\Theta$ em $S$; (iii) $\langle\Phi, \sigma\rangle-\Theta$ é consistente para todo $\langle\Phi, \sigma\rangle-\Theta$ em $S$.

\footnotetext{
${ }^{3} \operatorname{Notação:~} \operatorname{supp}(\operatorname{Perm}(\Upsilon)):=\cup_{\pi \in \operatorname{Perm}(\Upsilon)} \operatorname{supp}(\pi)$, onde $\operatorname{Perm}(\Upsilon):=\cup_{X \in \operatorname{Var}(\Upsilon)} \operatorname{perm}\left(\left.\Upsilon\right|_{X}\right)$.
} 
Teorema 4.1 (Teorema da Representação). Seja $\mathcal{P}_{\curlywedge}=\left\langle\Upsilon \vdash\left\{s_{1} \stackrel{\curlywedge}{\stackrel{?}{*}_{\alpha}} t_{1}, \ldots, s_{n}{\stackrel{\curlywedge}{{ }_{\alpha}}}_{\alpha}^{?} t_{n}\right\} \| \Omega \vdash\right.$ $\left.\left\{p_{1} \gtrless_{\alpha}^{?} q_{1}, \ldots, p_{m} \gtrless_{\alpha}^{?} q_{m}\right\}\right\rangle$ um problema de desunificação nominal. Defina a família $\Theta:=$ $\cup_{p_{i} \ddot{z}_{\alpha}^{?} q_{i} \in D_{\curlywedge}} \mathcal{U}\left(\Omega, p_{i} \stackrel{\curlywedge}{*}_{\alpha}^{?} q_{i}\right)$. Então o conjunto $S=\left\{\langle\Phi, \sigma\rangle-\Theta \mid\langle\Phi, \sigma\rangle \in \mathcal{U}\left(E_{\curlywedge}\right)\right.$ e $\Theta \not L$ $\langle\Phi, \sigma\rangle\}$ é uma representação completa de soluções para o problema $\mathcal{P}_{\curlywedge}$.

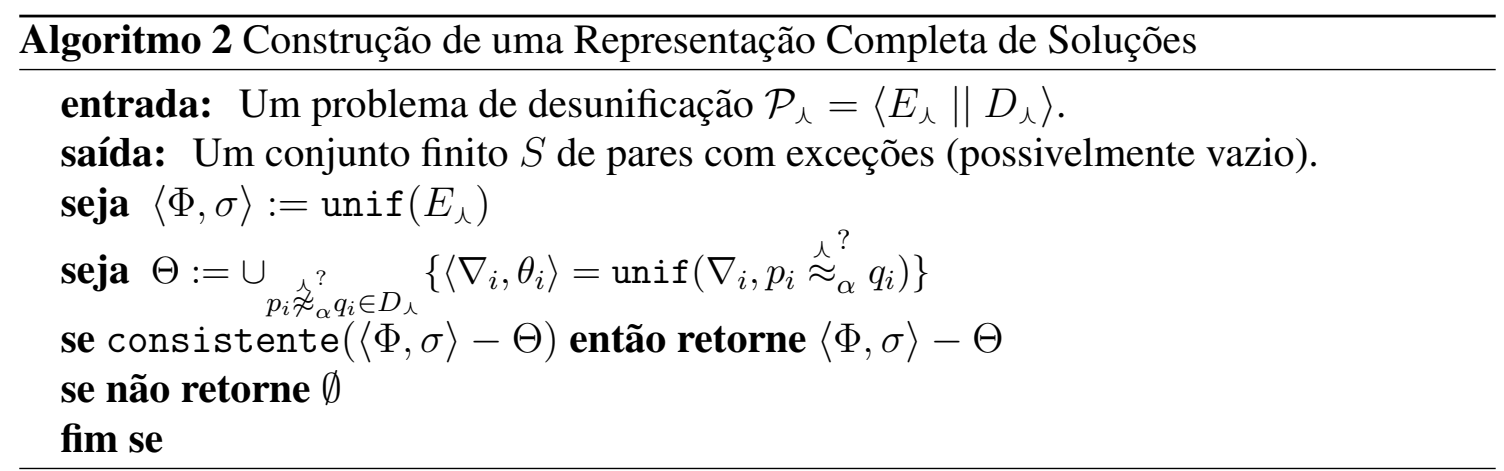

\section{Conclusão e Trabalhos Futuros}

Este trabalho fez uso da relação de ponto fixo, intrínseca à definição da relação de freshness, com o intuito de estender os conceitos da sintaxe já definidos na desunificação nominal usual. A abordagem via ponto fixo se mostrou útil para lidar com teorias equacionais que envolvem comutatividade justamente por considerar opções ignoradas sob a relação de freshness. Por isso, como trabalho futuro, pretendemos finalizar a análise semântica dessa nossa extensão e usufruir de sua representação finitária de soluções para investigar problemas envolvendo teorias equacionais.

\section{Referências}

Ayala-Rincón, M., de Carvalho Segundo, W., Fernández, M., and Nantes-Sobrinho, D. (2017). On solving nominal fixpoint equations. In Front. of Combining Systems - 11th Int. Symp., FroCoS 2017, Proc., volume 10483 of LNCS, pages 209-226. Springer.

Ayala-Rincón, M., Fernández, M., and Nantes-Sobrinho, D. (2020a). On nominal syntax and permutation fixed points. Log. Methods Comput. Sci., 16(1).

Ayala-Rincón, M., Fernández, M., Nantes-Sobrinho, D., and Vale, D. (2020b). On solving nominal disunification constraints. In Proc. of the 14th Work. on Logical and Semantic Frameworks with Applications, LSFA 2019, volume 348 of ENTCS, pages 3-22.

Buntine, W. L. and Bürckert, H. (1994). On solving equations and disequations. J. ACM, 41(4):591-629.

Comon, H. and Lescanne, P. (1989). Equational problems and disunification. J. Symb. Comput., 7(3/4):371-425.

Fernández, M. and Gabbay, M. (2007). Nominal rewriting. Inf. Comput., 205(6):917-965.

Gabbay, M. and Pitts, A. M. (2002). A new approach to abstract syntax with variable binding. Formal Aspects Comput., 13(3-5):341-363.

Pitts, A. M. (2013). Nominal Sets: Names and Symmetry in Computer Science. CUP.

Urban, C., Pitts, A. M., and Gabbay, M. (2004). Nominal unification. Theor. Comput. Sci., 323(1-3):473-497. 